\title{
On the radiation driven alignment of dust grains: Detection of the polarization hole in a starless core (Corrigendum)
}

\author{
F. O. Alves ${ }^{1}$, P. Frau ${ }^{2,3}$, J. M. Girart ${ }^{4}$, G. A. P. Franco ${ }^{5}$, F. P. Santos ${ }^{6}$, and H. Wiesemeyer ${ }^{7}$ \\ ${ }^{1}$ Max-Planck-Institut für extraterrestrische Physik, Giessenbachstrasse 1, 85748 Garching, Germany \\ e-mail: falves@mpe.mpg.de \\ 2 Instituto de Ciencia de Materiales de Madrid (CSIC), Sor Juana Inés de la Cruz 3, 28049 Madrid, Spain \\ 3 Observatorio Astronómico Nacional, Alfonso XII 3, 28014 Madrid, Spain \\ ${ }^{4}$ Institut de Ciències de l'Espai (CSIC-IEEC), Campus UAB, Facultat de Ciències, C5 par 2a 08193 Bellaterra, Catalunya, Spain \\ 5 Departamento de Física - ICEx - UFMG, Caixa Postal 702, 30.123-970 Belo Horizonte, Brazil \\ 6 Department of Physics and Astronomy, Northwestern University, 2145 Sheridan Road, Evanston, IL 60208, USA \\ 7 Max-Planck-Institut für Radioastronomie, Auf dem Hügel 69, 53121 Bonn, Germany
}

A\&A 569, L1 (2014), DOI: 10.1051/0004-6361/201424678

Key words. stars: formation - ISM: magnetic fields - techniques: polarimetric - ISM: individual objects: Core 109 - errata, addenda

Figure 2 in the original manuscript displays the polarization efficiency for the optical, near infrared and sub-millimeter (submm) polarimetric data as $P / A_{\mathrm{V}}$, where $P$ is the polarization degree and $A_{\mathrm{V}}$ is the visual extinction traced by the data. However, the polarization efficiency for the submm data should be simply $P$. By dividing $P$ by $A_{\mathrm{V}}$, we obtain a steeper and wrong slope for the decrease in polarization efficiency. The suitable form for Fig. 2 of the original document is exhibited in Fig. 1 of the present corrigendum. The submm polarization efficiency decreases as $\sim-1.0$ with respect to $A_{\mathrm{V}}$, instead of the originally published $\sim-2.0$.

Although the decrease in polarization efficiency at the submm regime is shallower than originally proposed, our modelling of the polarization degree with respect to the relative intensity, $I / I_{\max }$, still predicts the detection of a polarization hole (Sect. 3 of the published manuscript). Therefore, the main conclusions are unchanged.

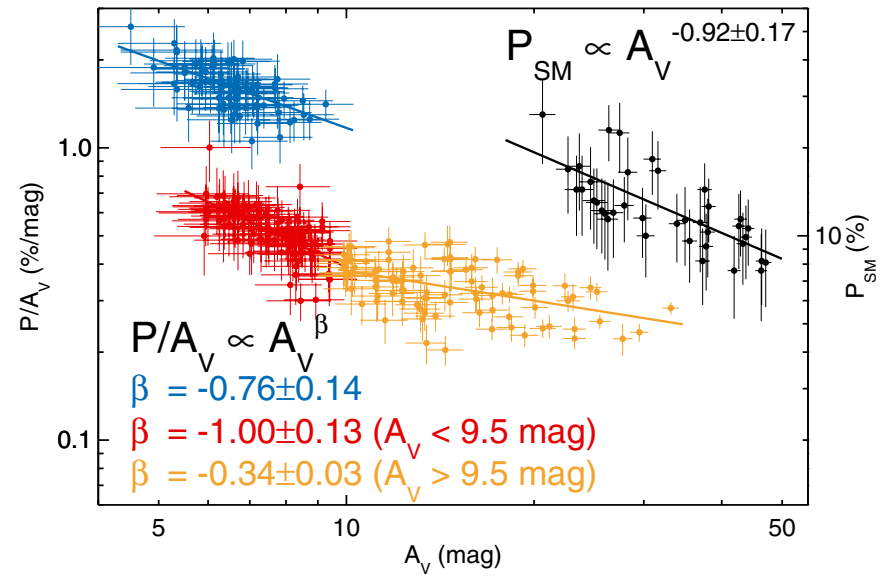

Fig. 1. Polarization efficiency of the optical and near-IR data $\left(P / A_{\mathrm{V}}\right)$ and the submm data $\left(P_{\mathrm{SM}}\right)$. 